\title{
Saturation Throughput Analysis of IEEE 802.11g (ERP-OFDM) Networks
}

\author{
Krzysztof Szczypiorski, Jozef Lubacz \\ Warsaw University of Technology, Institute of Telecommunications, \\ ul. Nowowiejska 15/19,00-665 Warsaw, Poland \\ $\{\mathrm{ksz}, j 1\}$ at.ele.pw.edu.pl
}

\begin{abstract}
This paper presents the saturation throughput analysis of IEEE 802.11g (ERP-OFDM) networks. The presented work is based on the Markov model previously introduced and validated by the authors in [7]. In the present paper the saturation throughput is evaluated in different channel conditions as a function of frame length.
\end{abstract}

Keywords: WLAN, IEEE 802.11, CSMA/CA, modeling

\section{Introduction}

The paper presents saturation throughput analysis of IEEE 802.11g (ERP-OFDM) networks. We use our general Markov-based model introduced and validated in [7]. The model presented in [7] is in line with the extensions of the basic Bianchi's model [1] which were proposed in [8] and [6]. The essential difference of the presented model with respect to the latter two is that it takes into account the effect of freezing of the stations' backoff timer along with the limitation of the number of retransmissions, maximum size of the contention window and the impact of transmission errors. The results presented in [7] showed that our model has good accuracy both in the case of error-free and error-prone channels. For both error-free and error-prone cases the proposed model shows better accuracy than the literature models with which it was compared (including: [1], [6] and [8]), especially for large number of stations.

The paper is organized as follows: Section 2 contains a summary of the model presented in [7]. Section 3 presents the saturation throughput analysis based on the given model. Finally, Section 4 presents essential conclusions.

\section{The Model}

We consider saturated conditions: stations have no empty queues and there is always a frame to be sent. $n$ stations compete for medium access (for $n=1$ only one station sends frames to other station which may only reply with ACK frame). Errors in the transmission medium are randomly distributed; this is the worst case for the frame

Please use the following format when citing this chapter:

Szczypiorski, K., Lubacz, J., 2007, in IFIP International Federation for Information Processing, Volume 245, Personal

Wireless Communications, eds. Simak, B., Bestak, R., Kozowska, E., (Boston: Springer), pp. 196-205. 
error rate-FER. All stations have the same bit error rate (BER). All stations are in transmission range and there are no hidden terminals. Stations communicate in ad hoc mode (BSS -- Basic Service Set) with basic access method. All stations use the same physical layer (PHY). The transmission data rate $R$ is the same and constant for all stations. All frames are of constant length $L$. Only data frames and ACK frames are exchanged. Collided frames are discarded -- the capture effect ([5]) is not considered.

The saturation throughput $S$ is defined as in [1]:

$$
S=\frac{E[D A T A]}{E[T]}
$$

where $\mathrm{E}[D A T A]$ is the mean value of the successfully transmitted payload, and $\mathrm{E}[T]$ is the mean value of the duration of the following channel states ([7]):

$T_{I}$ - idle slot,

$T_{S}$-- successful transmission,

$T_{C}$ - transmission with collision,

$T_{E D A T A}-$ unsuccessful transmission with data frame error,

$T_{E A C K}$ - unsuccessful transmission with ACK error.

Above channel states depend on:

$T_{P H Y h d r}$ - duration of a PLCP (PHY Layer Convergence Procedure) preamble and a PLCP header,

$T_{D A T A}$ - duration to transmit a data frame,

$T_{A C K}-$ duration to transmit an ACK frame,

$T_{\text {SIFS }} \cdots$ duration of SIFS (Short InterFrame Space),

$T_{\text {DIFS }}$ - duration of DIFS (DCF InterFrame Space),

$T_{E I F S}-$ duration of EIFS (Extended InterFrame Space).

The relation of the saturation throughput to physical channel characteristics is calculated similarly as in [6]:

$$
\left\{\begin{array}{l}
T_{l}=\sigma \\
T_{S}=2 T_{P H Y h d r}+T_{D A T A}+2 \delta+T_{S I F S}+T_{A C K}+T_{D I F S} \\
T_{C}=T_{P H Y h d r}+T_{D A T A}+\delta+T_{E I F S} \\
T_{E_{\text {.DATA }}}=T_{P H Y h d r}+\delta+T_{D A T A}+T_{E I F S} \\
T_{E_{-} A C K}=T_{S}
\end{array}\right.
$$

where $\sigma$ is the duration of the idle slot (aSlotTime [2]) and $\delta$ is the propagation delay.

For OFDM (Orthogonal Frequency Division Multiplexing) PHY, i.e. 802.11a [3] and $802.11 \mathrm{~g} \mathrm{[4]:}$

$$
\begin{aligned}
& T_{A C K}=T_{\text {Symbo }}\left[\begin{array}{l}
\frac{L_{S E R}+L_{T A I L}+L_{A C K}}{N_{B D S}} \\
T_{D A T A}=T_{\text {symbo }}
\end{array}\left[\frac{L_{S E R}+L_{T A I L}+L_{D A T A}}{N_{B D S}}\right\rceil\right.
\end{aligned}
$$

where:

$T_{\text {symbol }}$ - duration of a transmission symbol,

$L_{S E R}-$ OFDM PHY layer SERVICE field size,

$L_{T A L L}$ - OFDM PHY layer TAIL fields size, 
$N_{B p S}-$ number of encoded bits per one symbol,

$L_{A C K}-$ size of an ACK frame,

$L_{D A T A}$ - size of a data frame.

Values of $\sigma, T_{P H Y h d r}, T_{S I F S}, T_{D I F S}, T_{E I F S}, T_{s y m b o l}, N_{B p S}, L_{S E R}$, and $L_{T A I L}$ are defined in accordance with the 802.11 standard ([2], [3], or [4]).

Probabilities corresponding to states of the channel are denoted as follows:

$P_{I}$ - probability of idle slot,

$P_{S}$ - probability of successful transmission,

$P_{C}$ - probability of collision,

$P_{E D A T A}$ - probability of unsuccessful transmission due to data frame error,

$P_{E_{-} A C K}$ - probability of unsuccessful transmission due to ACK error.

Let $\tau$ be the probability of frame transmission, $p_{e_{-} \text {data }}$ the probability of data frame error and $p_{e} A C K$ the probability of $\mathrm{ACK}$ error. These are related to channel state probabilities as follows:

$$
\left\{\begin{array}{l}
P_{t}=(1-\tau)^{n} \\
P_{S}=n \tau(1-\tau)^{n-1}\left(1-p_{e_{-} \text {data }}\right)\left(1-p_{e_{-} A C K}\right) \\
P_{C}=1-(1-\tau)^{n}-n \tau(1-\tau)^{n-1} \\
P_{E_{-} D A T A}=n \tau(1-\tau)^{n-1} p_{e_{-} \text {data }} \\
P_{E_{-} A C K}=n \tau(1-\tau)^{n-1}\left(1-p_{e_{-} \text {data }}\right) p_{e_{-} A C K}
\end{array}\right.
$$

The saturation throughput $S$ equals

$$
S=\frac{P_{S} L_{p l d}}{T_{I} P_{1}+T_{S} P_{S}+T_{C}} \frac{P_{C}+T_{E_{-} D A T A} P_{E_{-} D A T A}+T_{E_{-} A C K} P_{E_{. .} A C K}}{}
$$

where $L_{\text {pld }}$ is MAC (Medium Access Control) payload size and $L_{p l d}=L-L_{\text {MAChdr, }}$ where $L_{M A C h d r}$ is the size of the MAC header plus the size of FCS (Frame Checksum Sequence).

$S$ can be normalized to data rate $R$ (called normalized $S$ ):

$$
\bar{S}=\frac{S}{R}
$$

where

$$
R=\frac{\mathrm{N}_{\mathrm{Bp} \mathrm{S}}}{T_{\text {symbol }}}
$$

Let $s(t)$ be a random variable describing DCF backoff stage at time $t$, with values from a set $\{0,1,2, \ldots, m\}$. Let $b(t)$ be a random variable describing the value of the backoff timer at time $t$, with values from a set $\left\{0,1,2, \ldots, W_{i}-1\right\}$. These random variables are dependent because the maximum value of the backoff timer depends on backoff stage:

$$
W_{i}= \begin{cases}2^{\prime} W_{0}, & i \leq m^{\prime} \\ 2^{2^{\prime}} W_{0}=W_{m}, & i>m^{\prime}\end{cases}
$$

where $W_{0}$ is an initial size of contention window and $m^{\prime}$ is a maximum number by 
which the contention window can be doubled; $m$ ' can be both greater and smaller than $m$ and also equal to $m$. $W_{0}$ and $W_{m}$ depend on $C W_{\min }$ and $C W_{\max }$ [2]:

$$
\begin{gathered}
W_{0}=C W_{\min }+1 \\
W_{m^{\prime}}=C W_{\max }+1=2^{m^{\prime}} W_{0}
\end{gathered}
$$

The two-dimensional process $(s(t), b(t))$ will be analyzed with an embedded Markov chain (in steady state) at time instants at which the channel state changes. Let $(i, k)$ denote the state of this process. The one-step conditional state transition probabilities will be denoted by $P=\left(\cdot, \cdot r_{,}\right)$.

Let $p_{f}$ be the probability of transmission failure and $p_{c o l l}$ the probability of collision. The non-null transition probabilities are determined as follows:

$$
\begin{cases}P(i, k \mid i, k+1)=1-p_{\text {coll }}, & 0 \leq i \leq m, 0 \leq k \leq W_{i}-2 \\ P(i, k \mid i, k)=p_{\text {coll }}, & 0 \leq i \leq m, 1 \leq k \leq W_{i}-1 \\ P(0, k \mid i, 0)=\left(1-p_{f}\right) / W_{0}, & 0 \leq i \leq m-1,0 \leq k \leq W_{0}-1 \\ P(i, k \mid i-1,0)=p_{f} / W_{i}, & 1 \leq i \leq m, 0 \leq k \leq W_{i}-1 \\ P(0, k \mid m, 0)=1 / W_{0}, & 0 \leq k \leq W_{0}-1\end{cases}
$$

Let $b_{i, k}$ be the probability of state $(i, k)$. It can be shown that:

$$
\begin{aligned}
& b_{i, 0}=p_{f} \cdot b_{i-1,0} \\
& b_{i, 0}=p_{f}^{i} \cdot b_{0,0}
\end{aligned}
$$

and

$$
b_{i, k}= \begin{cases}\frac{W_{i}-k}{W_{i}\left(1-p_{c o l l}\right)} p_{f}^{i} \cdot b_{0,0}, & 0<k \leq W_{i}-1 \\ p_{f}^{i} \cdot b_{0,0}, & k=0\end{cases}
$$

From:

$$
\sum_{i=0}^{m} \sum_{k=0}^{W_{j}-1} b_{i, k}=1
$$

And

$$
\sum_{i=0}^{m} b_{i, 0}=b_{0,0} \frac{1-p_{f}^{m+1}}{1-p_{f}}
$$

we get

$$
b_{0,0}^{-1}= \begin{cases}\frac{\left(1-p_{f}\right) W_{0}\left(1-\left(2 p_{f}\right)^{m+1}\right) \cdots\left(1-2 p_{f}\right)\left(1-p_{f}^{m+1}\right)}{2\left(1-2 p_{f}\right)\left(1-p_{f}\right)\left(1-p_{c o l l}\right)}+\frac{1-p_{f}^{m+1}}{1-p_{f}}, & m \leq m^{\prime} \\ \frac{\Psi}{2\left(1-2 p_{f}\right)\left(1-p_{f}\right)\left(1-p_{c s i l}\right)}+\frac{1-p_{f}^{m+1}}{1-p_{f}}, & m>m^{\prime}\end{cases}
$$

where

$$
\Psi=\left(1-p_{f}\right) W_{0}\left(1-\left(2 p_{f}\right)^{m^{\prime}+1}\right)-\left(1-2 p_{f}\right)\left(1-p_{f}^{m+1}\right)+W_{0} 2^{m^{\prime}} p_{f}^{m^{\prime}+1}\left(1-2 p_{f}\right)\left(1-p_{f}^{m-m^{i}}\right)
$$


The probability of frame transmission $\tau$ is equal to:

$$
\begin{gathered}
\tau=\sum_{i=0}^{m} b_{i, 0}= \\
= \begin{cases}\left(\frac{\left(1-p_{f}\right) W_{0}\left(1-\left(2 p_{f}\right)^{m+1}\right)-\left(1-2 p_{f}\right)\left(1-p_{f}{ }^{m+1}\right)}{2\left(1-2 p_{f}\right)\left(1-p_{f}\right)\left(1-p_{c o l l}\right)}+\frac{1-p_{f}^{m+1}}{1-p_{f}}\right)^{-1} \frac{1-p_{f}^{m+1}}{1-p_{f}}, & m \leq m^{\prime} \\
\frac{\Psi}{\left(\frac{1-p_{f}^{m+1}}{1-p_{f}}\right)^{-1} \frac{1-p_{f}^{m+!}}{1-p_{f}},\left(1-p_{f}\right)\left(1-p_{c o l l}\right)}+\frac{m>m^{\prime}}{1-p_{f}} & \end{cases}
\end{gathered}
$$

For $p_{\text {coll }}=0$ the above solution is the same as presented in [6].

The probability of transmission failure

$$
p_{f}=1-\left(1-p_{\text {coll }}\right)\left(1-p_{\varepsilon}\right)
$$

where $p_{e}$ is the frame error probability:

$$
p_{e}=1-\left(1-p_{e_{\text {dara }}}\right)\left(1-p_{e_{-} A C K}\right)
$$

where $p_{e_{\text {data }}}$ is FER for data frames and $p_{\varepsilon_{-} A C K}$ is FER for ACK frames. $p_{e_{-} \text {data }}$ and $p_{e_{-} A C K}$ can be calculated from bit error probability (i.e. BER) $p_{b}$ :

$$
\begin{aligned}
& p_{e_{-} \text {data }}=1-\left(1-p_{b}\right)^{L_{\text {data }}} \\
& p_{e_{-} A C K}=1-\left(1-p_{b}\right)^{L_{A C K}}
\end{aligned}
$$

The probability of collision

$$
p_{\text {coll }}=1-(1-\tau)^{n-1}
$$

Finally

$$
p_{f}=1-\left(1-p_{\text {coll }}\right)\left(1-p_{e}\right)=1-(1-\tau)^{n-1}\left(1-p_{e}\right)
$$

Equations (20) and (26) form a non-linear system with two unknown variables $\tau$ and $\mathrm{p}_{\mathrm{f}}$ which may be solved numerically.

\section{The Analysis}

All diagrams presented in this section show values of the normalized saturation throughput. All calculations were made for $n \in\{1,2,3,4,5,10,15,20,30,40\}$. For $L=1000$ bytes frame the following values of $B E R$ were used $\left\{10^{-4}, 5 \cdot 10^{-5}, 10^{-5}, 5 \cdot 10^{-6}\right.$, $\left.10^{-6}, 0\right\}$. For $L \in\{100,250,500,1000,1500,2000\}$ bytes $B E R \in\left\{0,10^{-5}, 10^{-4}\right\}$. We considered IEEE $802.1 \mathrm{lg}$ - ERP-OFDM i.e. "g" only mode and data rate $R=54 \mathrm{Mbps}$ (with the exception of the last diagram, which consists evaluation of $R$ impact on $S$ ).

Fig. 1 presents normalized $S$ as a function of $n$ for $L=1000$ bytes frame and different values of $B E R$ (Table 1). Along with increasing value of BER saturation throughput $S$ is reduced. Also maximum of $S$ is shifted from $n=2$ (for two smallest $B E R \quad 0$ and $10^{-6}$ ), through $n=3$ for $B E R 5 \cdot 10^{-6}$ and $10^{-5}, n=5$ and $5 \cdot 10^{-5}$, into $n=10$ for $B E R=10^{-4}$. Along with increasing value of BER presented curves are flattened. For a 
given $B E R$ reduction of $S$ with increase of $n$ is related to increasing number of collision in medium. Reduction of $S$ between $B E R=0$ and $B E R=10^{-6}$ is very small.

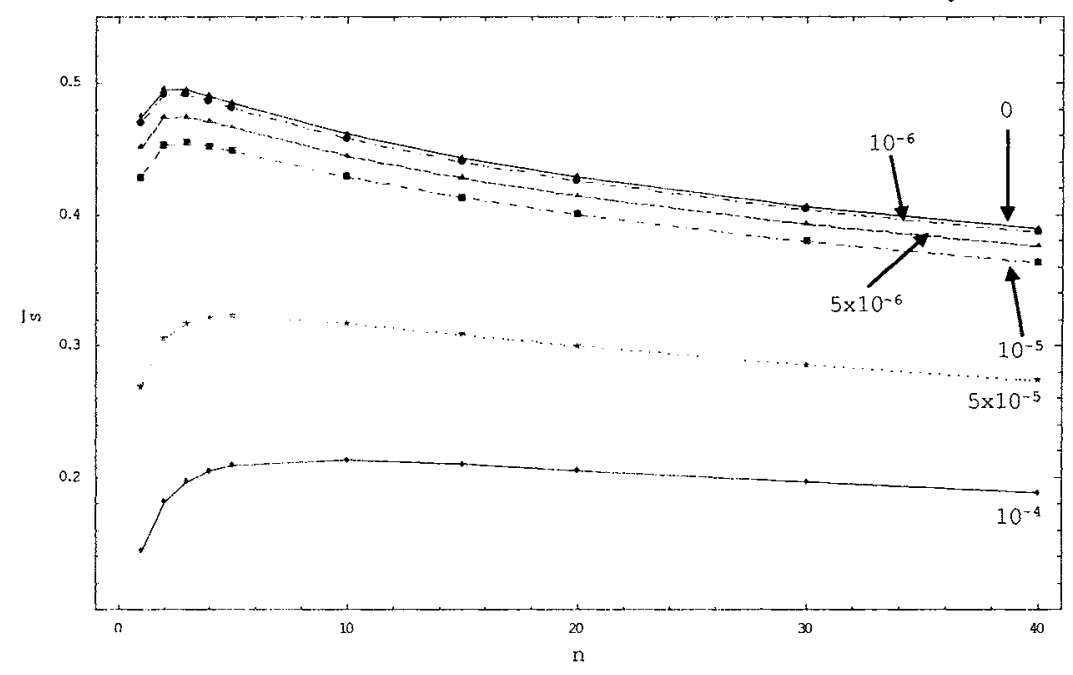

Fig. 1. Normalized $S$ as a function of $n$ - for $L=1000$ bytes and different values of $B E R$.

Fig. 2 presents normalized $S$ as a function of $n$ for different values of frame length and $B E R=0$ (Table 2). For a given $n$ along with increase of frame length the value of $S$ increases. Maximum value of $S$ depends on $n$ and falls into [2;5].

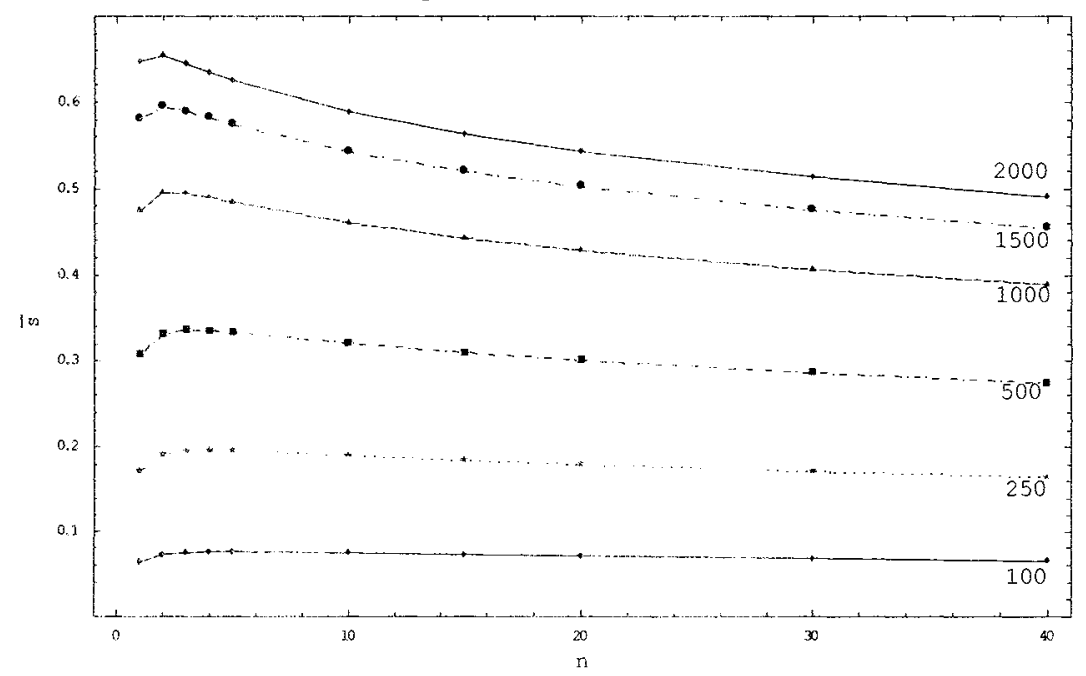

Fig. 2. Normalized $S$ as a function of $n$ - for different values of frame length and $B E R=0$.

Fig. 3 shows normalized $S$ as a function of $n$ for different values of frame length and $B E R=10^{-5}$ (Table 3). For a given $n$ along with increase of frame length the value 
of $S$ increases. Maximum value of $S$ depends on $n$ and falls into [2;5]. In comparison to $B E R=0 S$ is reduced because of channel errors.

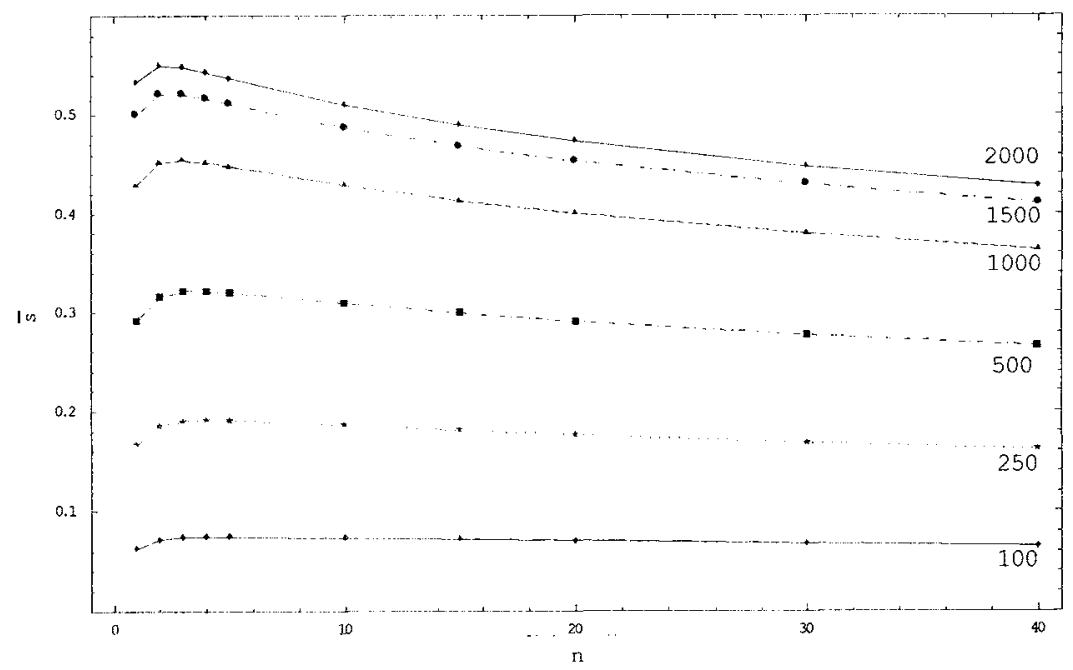

Fig. 3. Normalized $S$ as a function of $n$-for different values of frame length and $B E R=10^{-5}$.

Fig. 4 presents normalized $S$ as a function of $n$ for different values of frame length and $B E R=10^{-4}$ (Table 4). For a given $n$ along with increase of frame length the value of $S$ increases but only to the limited level; $S$ decreases for frames greater than 500 bytes and for $n \geq 3$. This is an influence of increasing FER with length of frame.

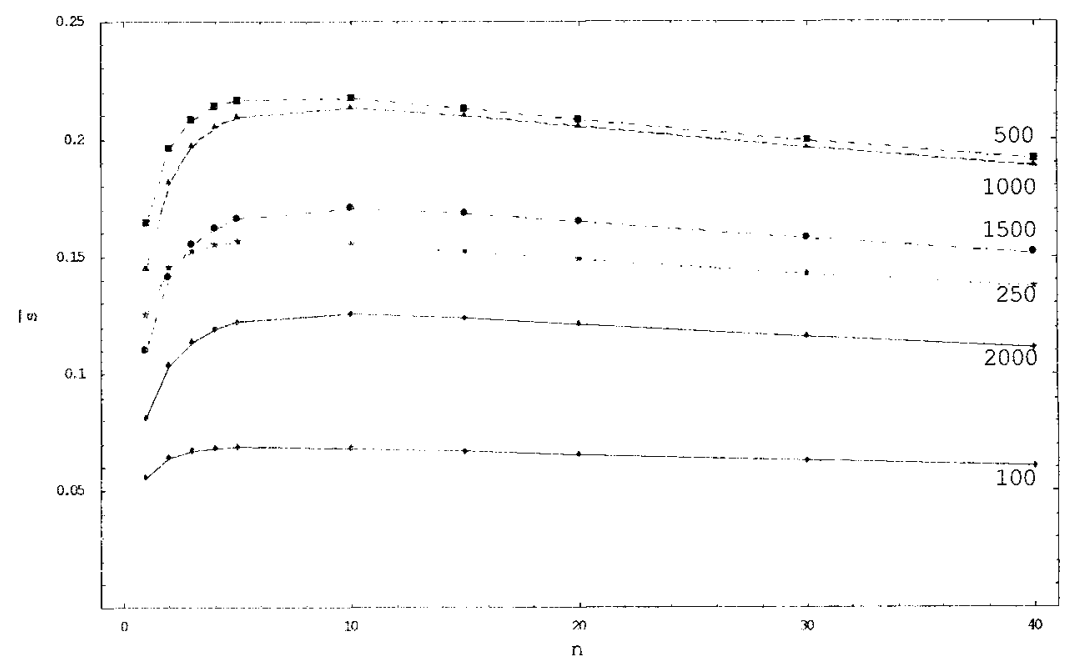

Fig. 4. Normalized $S$ as a function of $n-$ for different values of frame length and $B E R=10^{-4}$. 
Finally, we evaluate normalized $S$ as a function of $n$ for different IEEE $802.11 \mathrm{~g}$ data rates $R \in\{6,9,12,18,24,36,48,54\}$ Mbps. Results show (Fig. 5 and Table 5) that the channel usage for lower rates is better than for upper; for $6 \mathrm{Mbps}$ and $n=1$ is close to $85 \%$, while for $54 \mathrm{Mbps}$ and $n=1$ is close to $47 \%$.

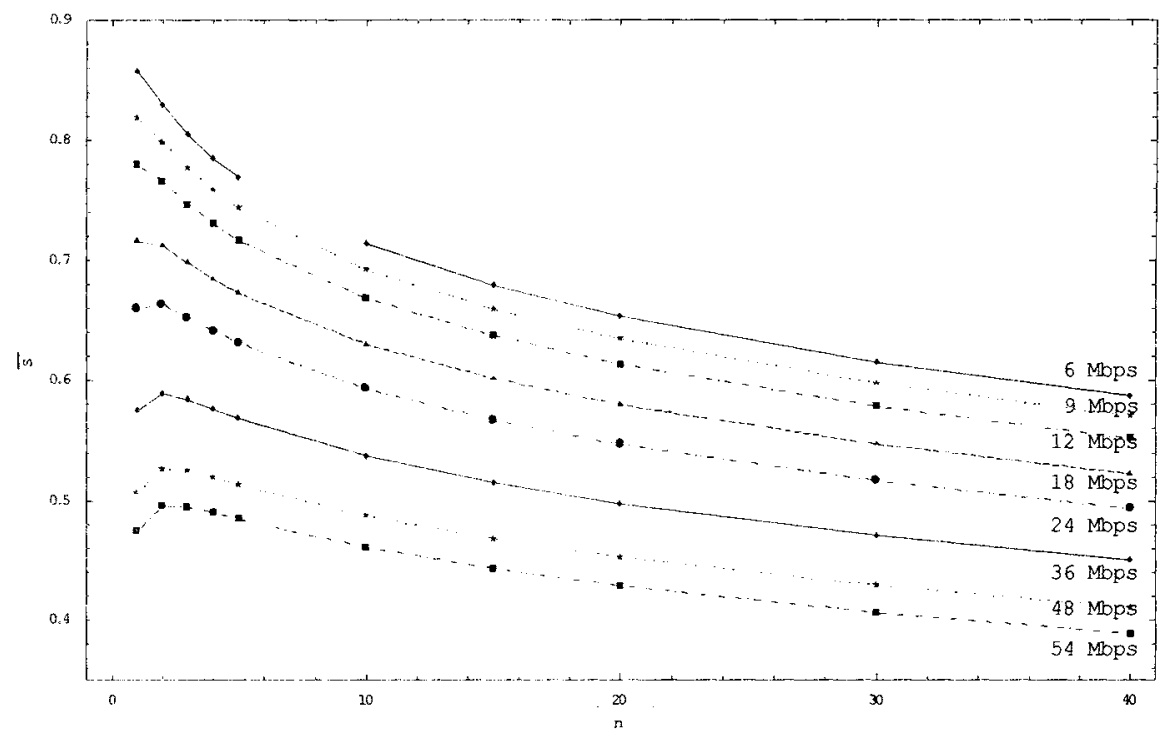

Fig. 5. Normalized $S$ as a function of $n$ - for $L=1000$ bytes, $B E R=0$, and different values of $R$.

Table 1. Normalized $S$ as a function of $n-$ for $L=1000$ bytes and different values of $B E R$.

\begin{tabular}{|c|c|c|c|c|c|c|}
\hline $\boldsymbol{B E R}$ & $\mathbf{1 0 ^ { - 4 }}$ & $\mathbf{5 \cdot 1 0 ^ { - 5 }}$ & $\mathbf{1 0 ^ { - 5 }}$ & $\mathbf{5 - 1 0 ^ { - 6 }}$ & $\mathbf{1 0 ^ { - 6 }}$ & \multicolumn{1}{c|}{$\mathbf{0}$} \\
\hline $\mathbf{1}$ & 0.1446 & 0.2688 & 0.4281 & 0.4510 & 0.4697 & 0.4745 \\
\hline $\mathbf{2}$ & 0.1816 & 0.3054 & 0.4521 & 0.4734 & 0.4909 & 0.4953 \\
\hline $\mathbf{3}$ & 0.1971 & 0.3170 & 0.4542 & 0.4740 & 0.4904 & 0.4945 \\
\hline $\mathbf{4}$ & 0.2050 & 0.3213 & 0.4517 & 0.4705 & 0.4860 & 0.4899 \\
\hline $\mathbf{5}$ & 0.2092 & 0.3226 & 0.4479 & 0.4660 & 0.4808 & 0.4845 \\
\hline $\mathbf{1 0}$ & 0.2131 & 0.3171 & 0.4285 & 0.4443 & 0.4574 & 0.4607 \\
\hline $\mathbf{1 5}$ & 0.2097 & 0.3082 & 0.4126 & 0.4275 & 0.4396 & 0.4427 \\
\hline $\mathbf{2 0}$ & 0.2052 & 0.2998 & 0.3997 & 0.4138 & 0.4254 & 0.4284 \\
\hline $\mathbf{3 0}$ & 0.1963 & 0.2853 & 0.3792 & 0.3925 & 0.4034 & 0.4061 \\
\hline $\mathbf{4 0}$ & 0.1885 & 0.2735 & 0.3631 & 0.3758 & 0.3863 & 0.3889 \\
\hline
\end{tabular}

Table 2. Normalized $S$ as a function of $n$-for different values of frame length and $B E R=0$.

\begin{tabular}{|c|c|c|c|c|c|c|}
\hline $\boldsymbol{n}$ & $\mathbf{1 0 0}$ & $\mathbf{2 5 0}$ & $\mathbf{5 0 0}$ & $\mathbf{1 0 0 0}$ & $\mathbf{1 5 0 0}$ & $\mathbf{2 0 0 0}$ \\
\hline $\mathbf{1}$ & 0.0637 & 0.1717 & 0.3074 & 0.4745 & 0.5808 & 0.6471 \\
\hline $\mathbf{2}$ & 0.0723 & 0.1904 & 0.3319 & 0.4953 & 0.5949 & 0.6541 \\
\hline $\mathbf{3}$ & 0.0748 & 0.1949 & 0.3360 & 0.4945 & 0.5896 & 0.6450 \\
\hline $\mathbf{4}$ & 0.0756 & 0.1958 & 0.3354 & 0.4899 & 0.5817 & 0.6346 \\
\hline $\mathbf{5}$ & 0.0758 & 0.1954 & 0.3334 & 0.4845 & 0.5738 & 0.6249 \\
\hline $\mathbf{1 0}$ & 0.0744 & 0.1899 & 0.3208 & 0.4607 & 0.5422 & 0.5880 \\
\hline
\end{tabular}




\begin{tabular}{|l|l|l|l|l|l|l|}
\hline \multicolumn{1}{|c|}{$\boldsymbol{n}$} & $\mathbf{1 0 0}$ & $\mathbf{2 5 0}$ & $\mathbf{5 0 0}$ & $\mathbf{1 0 0 0}$ & $\mathbf{1 5 0 0}$ & $\mathbf{2 0 0 0}$ \\
\hline $\mathbf{1 5}$ & 0.0725 & 0.1842 & 0.3098 & 0.4427 & 0.5197 & 0.5626 \\
\hline $\mathbf{2 0}$ & 0.0708 & 0.1793 & 0.3008 & 0.4284 & 0.5021 & 0.5430 \\
\hline $\mathbf{3 0}$ & 0.0678 & 0.1712 & 0.2862 & 0.4061 & 0.4751 & 0.5132 \\
\hline $\mathbf{4 0}$ & 0.0653 & 0.1646 & 0.2747 & 0.3889 & 0.4544 & 0.4905 \\
\hline
\end{tabular}

Table 3. Normalized $S$ as a function of $n$-for different values of frame length and $B E R=10^{-5}$.

\begin{tabular}{|c|c|c|c|c|c|c|}
\hline $\begin{array}{ll} & L\end{array}$ & 100 & 250 & 500 & 1000 & 1500 & 2000 \\
\hline 1 & 0.0629 & 0.1668 & 0.2909 & 0.4281 & 0.5004 & 0.5330 \\
\hline 2 & 0.0715 & 0.1855 & 0.3162 & 0.4521 & 0.5211 & 0.5502 \\
\hline 3 & 0.0740 & 0.1902 & 0.3212 & 0.4542 & 0.5208 & 0.5484 \\
\hline 4 & 0.0749 & 0.1914 & 0.3213 & 0.4517 & 0.5166 & 0.5431 \\
\hline 5 & 0.0751 & 0.1912 & 0.3199 & 0.4479 & 0.5114 & 0.5372 \\
\hline 10 & 0.0738 & 0.1862 & 0.3089 & 0.4285 & 0.4872 & 0.5105 \\
\hline 15 & 0.0719 & 0.1808 & 0.2988 & 0.4126 & 0.4682 & 0.4900 \\
\hline 20 & 0.0702 & 0.1760 & 0.2902 & 0.3997 & 0.4529 & 0.4736 \\
\hline 30 & 0.0673 & 0.1681 & 0.2763 & 0.3792 & 0.4289 & 0.4480 \\
\hline 40 & 0.0648 & 0.1617 & 0.2652 & 0.3631 & 0.4103 & 0.4281 \\
\hline
\end{tabular}

Table 4. Normalized $S$ as a function of $n$ - for different values of frame length and $B E R=10^{-4}$.

\begin{tabular}{|c|c|c|c|c|c|c|}
\hline $\begin{array}{ll} & L \\
n & \end{array}$ & 100 & 250 & 500 & 1000 & 1500 & 2000 \\
\hline 1 & 0.0556 & 0.1251 & 0.1643 & 0.1446 & 0.1103 & 0.0812 \\
\hline 2 & 0.0643 & 0.1450 & 0.1960 & 0.1816 & 0.1412 & 0.1038 \\
\hline 3 & 0.0671 & 0.1519 & 0.2081 & 0.1971 . & 0.1548 & 0.1137 \\
\hline 4 & 0.0682 & 0.1549 & 0.2137 & 0.2050 & 0.1619 & 0.1190 \\
\hline 5 & 0.0687 & 0.1561 & 0.2165 & 0.2092 & 0.1659 & 0.1219 \\
\hline 10 & 0.0682 & 0.1553 & 0.2172 & 0.2131 & 0.1705 & 0.1254 \\
\hline 15 & 0.0667 & 0.1520 & 0.2129 & 0.2097 & 0.1682 & 0.1237 \\
\hline 20 & 0.0652 & 0.1485 & 0.2081 & 0.2052 & 0.1647 & 0.1212 \\
\hline 30 & 0.0626 & 0.1423 & 0.1992 & 0.1963 & 0.1576 & 0.1159 \\
\hline 40 & 0.0604 & 0.1370 & 0.1916 & 0.1885 & 0.1512 & 0.1111 \\
\hline
\end{tabular}

Table 5. Normalized $S$ as a function of $n-$ for $L=1000$ bytes, $B E R=0$, and different values of $R$.

\begin{tabular}{|c|c|c|c|c|c|c|c|c|}
\hline $\boldsymbol{n}$ & $\mathbf{6}$ & $\mathbf{9}$ & $\mathbf{1 2}$ & $\mathbf{1 8}$ & $\mathbf{2 4}$ & $\mathbf{3 6}$ & $\mathbf{4 8}$ & $\mathbf{5 6}$ \\
\hline $\mathbf{1}$ & 0.8574 & 0.8186 & 0.7793 & 0.7158 & 0.6592 & 0.5752 & 0.5070 & 0.4745 \\
\hline $\mathbf{2}$ & 0.8290 & 0.7979 & 0.7651 & 0.7120 & 0.6629 & 0.5892 & 0.5267 & 0.4953 \\
\hline $\mathbf{3}$ & 0.8043 & 0.7762 & 0.7463 & 0.6978 & 0.6524 & 0.5840 & 0.5248 & 0.4945 \\
\hline $\mathbf{4}$ & 0.7844 & 0.7583 & 0.7301 & 0.6844 & 0.6412 & 0.5762 & 0.5193 & 0.4899 \\
\hline $\mathbf{5}$ & 0.7681 & 0.7432 & 0.7162 & 0.6725 & 0.6309 & 0.5684 & 0.5133 & 0.4845 \\
\hline $\mathbf{1 0}$ & 0.7136 & 0.6920 & 0.6682 & 0.6297 & 0.5928 & 0.5370 & 0.4872 & 0.4607 \\
\hline $\mathbf{1 5}$ & 0.6791 & 0.6591 & 0.6370 & 0.6013 & 0.5668 & 0.5147 & 0.4679 & 0.4427 \\
\hline $\mathbf{2 0}$ & 0.6534 & 0.6345 & 0.6135 & 0.5796 & 0.5468 & 0.4973 & 0.4526 & 0.4284 \\
\hline $\mathbf{3 0}$ & 0.6152 & 0.5978 & 0.5784 & 0.5470 & 0.5165 & 0.4706 & 0.4288 & 0.4061 \\
\hline $\mathbf{4 0}$ & 0.5867 & 0.5703 & 0.5520 & 0.5224 & 0.4936 & 0.4501 & 0.4105 & 0.3889 \\
\hline
\end{tabular}




\section{Conclusions}

The presented analysis shows that the saturation throughput essentially depends on bit error rate - for a given number of stations and the length of frame the lower $B E R$, the greater is $S$. This is an influence of channel error on the effective volume of transmitted data. Increasing the number of stations implies collisions and finally reduces the value of saturation throughput. The saturation throughput depends on frame error rate because $F E R$ is a function of $B E R$ and $L$.

\section{References}

1. Bianchi, G.: Performance Analysis of the IEEE 802.11 Distributed Coordination Function. IEEE Journal on Selected Areas in Communications, Vol. 18, No. 3 (2000) 535-547

2. IEEE 802.11, 1999 Edition (ISO/IEC 8802-11: 1999) IEEE Standards for Information Technology - Telecommunications and Information Exchange between Systems - Local and Metropolitan Area Network - Specific Requirements - Part 11: Wireless LAN Medium Access Control (MAC) and Physical Layer (PHY) Specifications (1999)

3. IEEE 802.11a-1999 (8802-11:1999/Amd 1:2000(E)), IEEE Standard for Information technology - Telecommunications and information exchange between systems - Local and metropolitan area networks - Specific requirements - Part 11: Wireless LAN Medium Access Control (MAC) and Physical Layer (PHY) specifications - Amendment 1: Highspeed Physical Layer in the $5 \mathrm{GHz}$ band (1999)

4. IEEE 802.11g-2003 IEEE Standard for Information technology - Telecommunications and information exchange between systems - Local and metropolitan area networks Specific requirements -- Part 11: Wireless LAN Medium Access Control (MAC) and Physical Layer (PHY) specifications - Amendment 4: Further Higher-Speed Physical Layer Extension in the $2.4 \mathrm{GHz}$ Band (2003)

5. Kochut, A., Vasan, A., Shankar, A., Agrawala, A.: Sniffing Out the Correct Physical Layer Capture Model in 802.11b. In: 12th IEEE International Conference on Network Protocols (ICNP 2004), Berlin (2004)

6. Ni, Q., Li, T., Turletti, T., Xiao, Y.: Saturation Throughput Analysis of Error-Prone 802.11 Wireless Networks. Wiley Journal of Wireless Communications and Mobile Computing (JWCMC), Vol. 5, Issue 8 (2005) 945-956

7. Szczypiorski, K., Lubacz, J.: Performance Evaluation of IEEE 802.11 DCF Networks. In: 20th International Teletraffic Congress (ITC-20), Ottawa, Canada, June 17-21, 2007; Lecture Notes in Computer Science (LNCS) 4516, Springer-Verlag Berlin Heidelberg (2007) 1082-1093

8. Wu, H., Peng, Y., Long, K., Cheng, S., Ma, J.: Performance of Reliable Transport Protocol over IEEE 802.11 Wireless LAN: Analysis and Enhancement. In: IEEE INFOCOMO2 (2002) 